\title{
Effectively Indexing the Multi-Dimensional Uncertain Objects for Range Searching
}

\author{
Ying Zhang, Wenjie Zhang, Qianlu Lin, Xuemin Lin* \\ The University Of New South Wales \\ \{yingz, zhangw, qlin, lxue \}@cse.unsw.edu.au
}

\begin{abstract}
The range searching problem is fundamental in a wide spectrum of applications such as radio frequency identification (RFID) networks, location based services (LBS), and global position system (GPS). As the uncertainty is inherent in those applications, it is highly demanded to address the uncertainty in the range search since the traditional techniques cannot be applied due to the inherence difference between the uncertain data and traditional data. In the paper, we propose a novel indexing structure, named $U$-Quadtree, to organize the uncertain objects in a multi-dimensional space such that the range searching can be answered efficiently by applying filtering techniques. Particularly, based on some insights of the range search on uncertain data, we propose a cost model which carefully considers various factors that may impact the performance of the range searching. Then an effective and efficient index construction algorithm is proposed to build the optimal $U$-Quadtree regarding the cost model. Comprehensive experiments demonstrate that our technique outperforms the existing works for range searching on multi-dimensional uncertain objects.
\end{abstract}

\section{Categories and Subject Descriptors}

H.2.8 [Database Applications]: Spatial databases and GIS

\section{General Terms}

Algorithm, Performance

\section{Keywords}

Indexing, mutidimensional uncertain objects, range query

\section{INTRODUCTION}

In recent years, the database community has witnessed the increasing amount of research on uncertain data modeling

\footnotetext{
* Corresponding Author
}

Permission to make digital or hard copies of all or part of this work for personal or classroom use is granted without fee provided that copies are not made or distributed for profit or commercial advantage and that copies bear this notice and the full citation on the first page. To copy otherwise, to republish, to post on servers or to redistribute to lists, requires prior specific permission and/or a fee.

EDBT 2012, March 26-30, 2012, Berlin, Germany.

Copyright 2012 ACM 978-1-4503-0790-1/12/03 ...\$10.00 and processing, due to its importance in many important applications such as data cleaning, radio frequency identification (RFID) networks, location based services (LBS), global position system (GPS), sensor data analysis, economic decision making and market surveillance. Common causes of uncertainty in these applications include data randomness and incompleteness, limitation of measuring equipment, delay or loss of data updates and privacy preservation.

The range searching problem is fundamental in the above applications. Below are two examples.

In some warehouse management systems, the RFID tags are attached to the items and their current locations can be obtained by RFID readers. Since the RFID reading may be noisy due to the sensitivity of the low cost readers to various environmental factors such as interference from nearby mental objects and contention among tags [23], the location of an object is modeled as a multi-dimensional uncertain object as shown in Fig 1, where an object $A$ is represented by an uncertain region $A_{r}$ and the probabilistic density function $(P D F)$ A.pdf. This implies that an object may appear at the locations within its uncertain region with probabilities described by its $P D F$. In some scenerios, a manager of the warehouse may want to identify the objects within a search region e.g., the objects covered by a fire sprinkler (the circular region $r_{q}$ in Fig. 1) or the objects within a particular stock keeping area. As shown in Fig. 1, it is meaningless to tell if the object $A$ appears within search region $r_{q}$ or not. Instead, the notation of appearance probability is proposed in [21] to capture the likelihood of $A$ falling in $r_{q}$. As the manager may only be interested in the objects with a sufficiently large chance to fall in the search region, the system will report the objects with probability at least $\theta$ appearing in $r_{q}$ where the probabilistic threshold $\theta$ is determined by the manager.

Another example application of range search on uncertain data is the location based services (LBS). The location of a mobile user can be described as an uncertain object, because the location may be derived based on the nearest contour lines of user's possible location regarding the nearby towers [4]. Based on the user's location information, the range search can help a supermarket to send advertising coupons via SMS to potential customers who are likely to be in the vicinity of the supermarket [19].

Motivated by the above applications, in the paper we study the problem of probabilistic threshold range query on multi-dimensional uncertain data; that is, given a set $\mathcal{U}$ of multi-dimensional uncertain objects and a search region $r_{q}$, report the objects with appearance probability at least $\theta$. This is formally defined in Section 2.

Challenges. A straightforward approach for this problem 


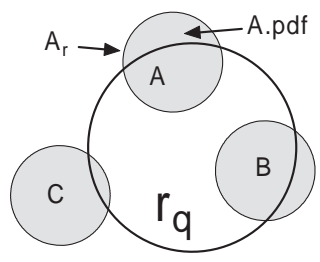

Figure 1: Example of range search on uncertain objects

is to calculate the appearance probability of each individual object and then report the ones whose appearance probabilities exceed the given probabilistic threshold. This is cost-inhibitive because the computation of the appearance probability may be very expensive because of the integral calculation or I/O costs involved. Therefore, all of the existing works $[16,20,21,25]$ on range search against multidimensional uncertain data with arbitrary $P D F$ follow the filtering-and-verification paradigm such that, with the help of the indexing structures, many objects are filtered at a reasonable filtering cost without explicit calculation of their appearance probabilities. The key idea of the existing techniques is as follows: a summary (See details in Section 2.2 and Section 3.1) of the PDF is pre-computed for each uncertain object to approximately capture the distribution of its $P D F$, and the summaries are organized by augmenting existing index techniques (e.g., $R$-Tree). For a given search region $r_{q}$, the lower and upper bounds of the appearance probability can be derived at a cheap cost for each uncertain object. Then an uncertain object $U$ may be filtered in two ways: $(i) U$ is pruned if the upper bound of the appearance probability is smaller than the given probabilistic threshold $\theta$, or (ii) $U$ is validated (qualified) if the lower bound of the appearance probability is not less than $\theta$. Only the objects survived the filtering phase need to be verified, i.e., explictly computing their appearance probabilities.

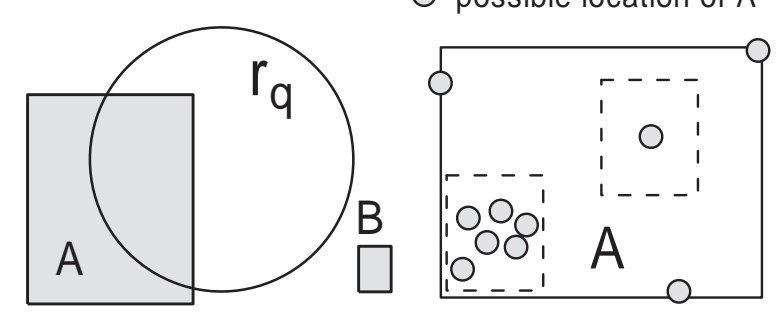

Figure 2: Region size Figure 3: Diff. Densities

Motivation. Intuitively, the more resources (i.e., larger summary size in terms of space usage) assigned to the summary of an uncertain object $U$, the tighter the lower and upper appearance probability bounds of $U$ and hence the lower expected verification cost of $U$. On the other hand, the filtering cost, which is usually dominated by the index $\mathrm{I} / \mathrm{O}$ delay, increases with the storage overhead of the summaries. Therefore, the key of the index construction is to find a good trade-off between filtering cost and verification cost such that the overall cost is minimized. Based on the above observations, following are three important factors which may affect the resource allocation policies of the summaries during the index construction.

(i) Uncertain region size. Suppose there are two uncertain objects $A$ and $B$ as shown in Fig. 2 where the uncertain region size of $A$ is much larger than that of $B$, intuitively we should allocate more resources to the $P D F$ summary of $A$ because, with much higher chance, $A$ will survive the filtering phase and hence invoke verification cost. In an extreme case when the uncertain region of $B$ is a point, it is meaningless to assign extra resources to $B$ besides the location of the point.

(ii) Verification cost. The verification costs of the uncertain objects may vary due to different $P D F s$ and I/O costs involved. Intuitively, more resources should be allocated to the objects with higher verification costs. Moveover, even if all objects have the same verification cost, the ratio of the index I/O delay and the verification cost per object should be considered. This is because the higher the verification cost per object, the more expensive filtering cost (i.e., larger index size) we can afford since the gain of a successful validation/pruning is more significant.

(iii) Density of the PDF. As depicted in Fig. 3, the distribution of the possible locations of an uncertain object $A$ may be uneven. Regarding the resource allocation for a particular uncertain object, intuitively the dense part of the object should have the priority because it contributes more to the appearance probability than the sparse part. This implies that we should carefully consider the density when building the summary of an uncertain object.

As discussed in Section 2, some of the existing works [21, $25]$ do consider the density factor during the index construction. However, the factors of uncertain region size and verification cost are neglected. This is because the same amount of resources in terms of the space usage are allocated to the summary of each uncertain object in the existing works. Consequently, this "equality strategy" may inherently limit the performance of the indexing techniques.

Motivated by these facts, in the paper we propose an effective and efficient indexing technique for the range search against the uncertain objects, in which above factors are carefully considered according to our quantitative analysis of the cost model for the range search. Our contributions can be summarized as follows.

- To facilitate the range search, we propose a novel technique called $U$-Quadtree to effectively index the multidimensional uncertain objects with arbitrary PDFs.

- Assume that the query distribution is known, we propose a cost model to quantitatively analyze the performance of the range search. Then we develop an efficient optimal $U$-Quadtree construction algorithm guided by the cost model. We also discuss how to construct the $U$-Quadtree when the query distribution is unknown.

- Comprehensive experiments demonstrate the efficiency of the $U$-Quadtree technique.

The remainder of the paper is organized as follows. We formally define the problem of range search and introduce the related work in Section 2. Section 3 presents the $U$ Quadtree structure and range search algorithm, followed by a cost model. Section 4 develops an optimal $U$-Quadtree construction algorithm based on the cost model. Results of comprehensive performance studies are presented in Section 5. Finally, Section 6 concludes the paper.

\section{PRELIMINARY}

In this section, we first formally define the problem of range search on multi-dimensional uncertain objects. Then 
we introduce the related works. Table 1 summaries notations frequently used throughout the paper.

\begin{tabular}{|c|l|}
\hline Notation & Definition \\
\hline \hline$U(\mathcal{U})$ & uncertain object (a set of uncertain objects) \\
\hline$n$ & the number of uncertain objects \\
\hline$r_{q}$ & range search region \\
\hline$P_{a p p}\left(U, r_{q}\right)$ & the appearance probability of $U$ regarding $r_{q}$ \\
\hline$\theta$ & probabilistic threshold \\
\hline$c, l(c)$ & a cell of the $U$-Quadtree, the level of $c$ \\
\hline$h$ & the height of the $U$-Quadtree \\
\hline$f$ & entry page capacity \\
\hline$\varphi(c)$ & cell $c$ and its descendent cells \\
\hline$\chi(c)$ & child cells of the cell $c$ \\
\hline$x \in c$ & instance $x$ is contained by cell $c$ \\
\hline$t_{U}$ & the verification cost of $U$ \\
\hline$P_{c}(c)\left(P_{o}(c)\right)$ & containing (overlapping) probability of $c$ \\
\hline$F_{c}\left(V_{c}\right)$ & the filtering (verification) factor of $c$ \\
\hline$S_{U}, S_{U}^{*}$ & summary of $U$, optimal summary of $U$ \\
\hline$\omega\left(S_{U}\right)$ & the cost of the summary $S_{U}$ \\
\hline$\omega(e)$ & the cost of the entry $e$ \\
\hline$x \uparrow S_{U}(c)$ & instance $x$ is assigned to cell $c$ in $S_{U}$ \\
\hline
\end{tabular}

Table 1: The summary of notations.

\subsection{Problem Definition}

A point (instance) $x$ referred in the paper, by default, is in a $d$-dimensional numerical space. In the paper, an uncertain object is represented by its possible locations (points) and the probability it may appear at each location. Particularly, an uncertain object can be described either continuously or discretely. In the continuous case, an uncertain object $U$ is described by its probability density function(PDF), denoted by $U . p d f$, and its uncertain region $U_{r}$. The probability of $U$ appearing at location $x$ is $U \cdot p d f(x)$ and we have $\int_{x \in U_{r}} U . p d f(x) d x=1$. Note that we assume PDFs of the uncertain objects are mutually independent and various objects may have different $P D F s$ and uncertain regions. In some applications, the $P D F$ of the uncertain object may not be available and hence an uncertain object is represented by a set of sampled points (discrete case); that is, an uncertain object consists of a set of instances (points) $u_{1}, u_{2}$, $\ldots, u_{m}$, where $U$ occurs at location $u_{i}$ with probability $u_{i p}$ and $\sum_{u \in U} u_{p}=1$. For presentation simplicity, the "object" referred in the rest of the paper is the "multi-dimensional uncertain object" unless otherwise specified.

In the paper, $U_{m b b}$ denotes the minimal bounding box (MBB) of the instances of an object $U$. For a point $p$ and a region $r, p \in r$ means that $p$ is contained by $r$. For any two regions $r_{1}$ and $r_{2}, r_{1}$ contains $r_{2}$ ( $r_{2}$ is contained by $\left.r_{1}\right)$, denoted by $r_{2} \subseteq r_{1}$, if $r_{1} \cup r_{2}=r_{1}$. We say $r_{1}$ overlaps $r_{2}$ ( $r_{2}$ is overlapped by $r_{1}$ ) if $r_{2} \not r_{1}$ and $r_{1} \cap r_{2} \neq \emptyset$.

For a given search region $r_{q}$, we use $P_{a p p}\left(U, r_{q}\right)$ to represent the likelihood of $U$ falling in $r_{q}$, called appearance probability of $U$ regarding $r_{q}$, which is formally defined below.

For continuous cases,

$$
P_{a p p}\left(U, r_{q}\right)=\int_{x \in U_{r} \cap r_{q}} U \cdot p d f(x) d x
$$

For discrete cases,

$$
P_{a p p}\left(U, r_{q}\right)=\sum_{u \in U \wedge u \in r_{q}} u_{p}
$$

For presentation simplicity, we concentrate on the discrete case in the paper. Nevertheless, all techniques developed in the paper can be immediately applied to continuous case.

Below is the definition of "probabilistic threshold range query", which is abbreviated to "range query" in the rest of the paper.

Definition 1. Probabilistic Threshold Range Query. Given a set $\mathcal{U}$ of uncertain objects and a search region $r_{q}$, the probabilistic threshold range query retrieves all objects $U \in \mathcal{U}$ with $P_{a p p}\left(U, r_{q}\right) \geq \theta$ where $\theta(0<\theta \leq 1)$ is the user specified probabilistic threshold.

EXAMPLE 1. In Fig 4, suppose objects have four instances each and all instances have the same occurrence probability (0.25). According to Equation 2, we have $P_{a p p}\left(A, r_{q}\right)=0.5$, $P_{a p p}\left(B, r_{q}\right)=0.75$ and $P_{a p p}\left(C, r_{q}\right)=0.25$. For the given range query $q$ with search region $r_{q}$ and the probabilistic threshold 0.5, objects $A$ and $B$ will be reported.

\section{Problem Statement.}

In this paper we investigate the problem of probabilistic threshold range query over multi-dimensional uncertain objects with arbitrary PDFs. Particularly, we aim to develop effective indexing mechanism to facilitate the range query processing.

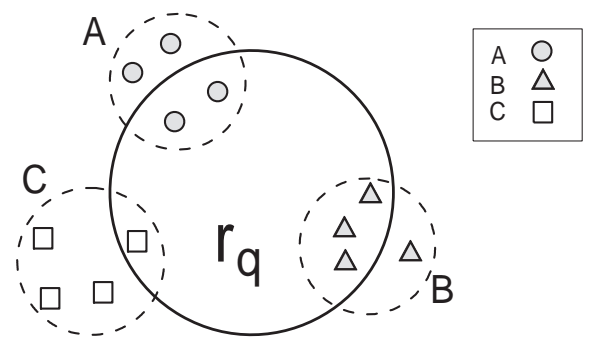

Figure 4: Appearance Probability

\subsection{Related Work}

In this subsection, we first present four indexing techniques supporting range search on multi-dimensional uncertain objects with arbitrary PDFs, including $R$-Tree, $U$ Tree, UI-Tree and UP-Index. Then we briefly introduce other related indexing techniques for uncertain data.

$R$-Tree. In $R$-Tree approach [16, 20, 17], the MBB of an object serves as the summary of its $P D F$, and MBBs are organized by R-Tree. An uncertain object $U$ can be validated if its $\mathrm{MBB}$ is contained by $r_{q}$, i.e., $U_{m b b} \subseteq r_{q}$, regardless of the value of the probabilistic threshold $\theta$. Similarly, $U$ is pruned if $U_{m b b}$ does not intersect $r_{q}$, i.e., $U_{m b b} \cap r_{q}=\emptyset$. This approach is simple and performs well if the uncertain region sizes are much smaller than $r_{q}$. However, as the MBB cannot further explore the $P D F$ of an uncertain object, the filtering capacity of the index is poor when the size of the uncertain region is not small. As shown in Fig. 5, for a given search region $r_{q}$, we cannot prune $A$ regarding any probabilistic threshold $\theta$ although intuitively $P_{a p p}\left(A, r_{q}\right)$ should be small. Similarly, $B$ cannot be validated either.

$U$-Tree. The PDF summary of an object in $U$-Tree is a finite set of probabilistically constrained regions (PCRs), which is introduced by Tao et al. in [21]. PCR is a general version of $x$-bounds which aims to index one dimensional uncertain data [9]. For a given $\theta(0 \leq \theta \leq 0.5)$, the $P C R$ of an object $U$ regarding $\theta$, denoted by $U \cdot p c r(\theta)$, is constructed as 
follows. As shown in Fig. 6, in each dimension, two lines are calculated. In the horizontal dimension, $U$ has the probability $\theta$ to occur on the left side of line $l_{1-}$, also probability $\theta$ to occur on the right side of line $l_{1+}$. Similarly, $l_{2-}$ and $l_{2+}$ are calculated in the vertical dimension. The shaded region in Fig. 6 is the geometric representation of $\operatorname{U} . p c r(\theta)$. Then we can take advantage of $U \cdot \operatorname{pcr}(\theta)$ to prune or validate $U$ regarding $\theta$ and $r_{q}$. For instance, as shown in Fig. 6, suppose $\theta$ is the probabilistic threshold for two search regions $r_{q}^{1}$ and $r_{q}^{2}$. $U$ can be pruned regarding $r_{q}^{1}$ because $r_{q}^{1}$ does not intersect $U \cdot p \operatorname{cr}(\theta)$. On the other hand, $U$ can be validated with respect to $r_{q}^{2}$ since all instances below $l_{2}$ are contained by $r_{q}^{2}$. As it is infeasible to keep all $\operatorname{U} p \operatorname{cr}(\theta)$ for any $\theta \in[0,0.5]$, a finite number of $P C R$ s are pre-computed for each object and the lower and upper appearance probability bounds can be derived. Based on the PCRs of the uncertain objects, $U$-Tree is built up in a similar way with $R$-Tree where each entry in a leaf node corresponds to the PCRs of an uncertain object.

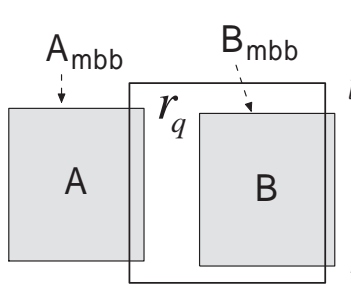

Figure 5: MBB

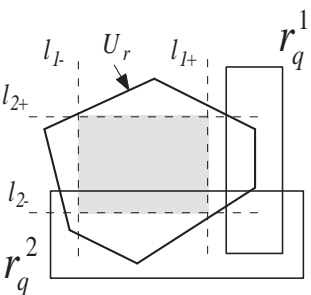

Figure 6: PCR
$U I$-Tree. The PDF summary of an object $U$ in [25] is a set of groups which are disjointed partitions of its $P D F$ based on a $K D$-Tree. Given a search region $r_{q}$, we can derive the lower and upper bounds of $P_{a p p}\left(U, r_{q}\right)$ based on the topological relationships between the groups and $r_{q}$. Specifically, groups contained by $r_{q}$ contribute to both lower and upper bounds of the appearance probability since all instances in these groups are contained by $r_{q}$. With similar rationale, groups overlapped by $r_{q}$ only contribute to upper bound. Then an object $U$ may be validated (pruned) based on the lower (upper) bound of $P_{a p p}\left(U, r_{q}\right)$. For the space efficiency, the groups of the uncertain objects may be merged such that a set of groups from different objects can share the same boundary, namely "word" in [25]. The identifications of the related objects and their corresponding probability mass are kept in each "word". Then UI-Tree is constructed in a similarly way with $R$-Tree where each entry of a leaf node is a "word".

UP-Index. Recently, Angiulli et al. [3] develop a pivot based indexing mechanism for uncertain data in general metric space. For a given pivot point $p$ and an object $U$, the $P D F$ summary of $U$ is the histogram of the distance distribution regarding $p$ and $U$. The upper bound of $P_{a p p}\left(U, r_{q}\right)$ can be derived based on the reverse triangle inequality according to the histogram and the distance between the centre of $r_{q}$ and the pivot point $p$. Then an object can be pruned based on the upper bound derived. To enhance the pruning power, a set of pivot points are employed in [3]. The advantage of $U P$-Index is that it can support distance based range query in general metric space. Nevertheless, as shown in our empirical study, its performance is not competitive under our problem setting because : $(i)$ an object cannot be validated in [3] because $U P$-Index cannot derive the lower bound of $P_{a p p}\left(U, r_{q}\right)$, and (ii) for any range search the whole index is scanned to prune objects, and the index size is usually large for a decent pruning capacity.

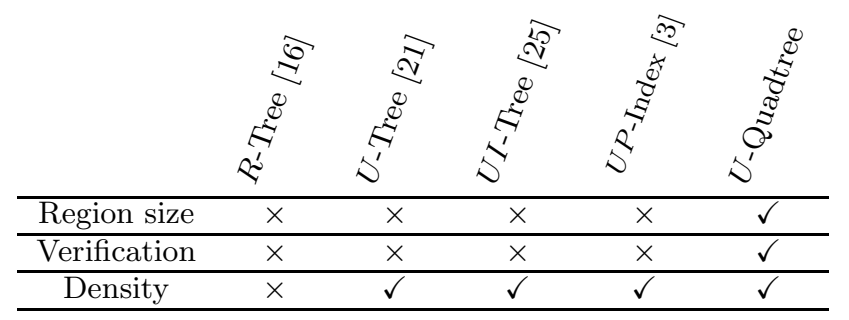

Table 2: Summary of the indexing techniques

Table 2 illustrates the properties of the indexing techniques regarding three factors mentioned in Section 1. Clearly, $R$-Tree based approach considers none of the factors since only one MBB is allocated for the PDF summary of each object. $U$-Tree, $U I$-Tree and $U P$-Index techniques consider the density factor because they attempt to evenly distribute the accumulated probabilities in the summary of an object $U$, which implies that more resources are allocated for the dense parts of $U$. However, the same amount of resources in terms of the space usage are allocated to the summary of each object in these techniques. Consequently, they cannot effectively address different uncertain region sizes and verification costs during the index construction. In Section 4, we show that $U$-Quadtree technique proposed in the paper carefully considers all three factors.

Others. There are also some studies on indexing multidimensional uncertain objects which focus on specific cases of objects' PDFs and queries. For instances, in $[5,6,8$, 13], Böhm et al. study range queries with the constraint that PDFs of uncertain objects follow Gaussian or uniform distributions. Assuming PDFs of the objects are either histograms or more complex ones such as Gaussian or piecewise algebraic, in [1] Agarwal et al. provide thorough theoretical analysis on range search on uncertain data. Managing uncertain moving objects [24] and uncertain categorical data [20] have been separately studied. Aggarwal et al. [2] study the problem of indexing high dimensional uncertain data with the assumption that the PDFs of the uncertain object on each dimension are independent to others. Assuming the space is partitioned by a virtual grid with limited number of cells, Ma et al. [18] propose solutions for efficient retrieval of uncertain spatial point data where the location information is derived from the free text by spatial expressions. Recently, Kinura et al. [15] propose a primary indexing technique named UPI to speed up the query processing on uncertain data by clustering the heap files, in which $U$-Tree [21] technique is used as a building block to index uncertain objects with arbitrary PDFs. In [17], Lian et al. propose a generic framework to index uncertain data. Their main focus is how to tackle the local correlations among uncertain objects, and their indexing technique falls in the $R$-Tree category.

\section{U-QUADTREE}

In the paper, our index structure is based on the quadtree because it is a flexible data structure in the sense that we can adaptively build summaries of the objects so that the overall cost of range searching can be minimized regarding the cost model proposed in the paper. Section 3.1 formally introduces $U$-Quadtree structure, and Section 3.2 presents 


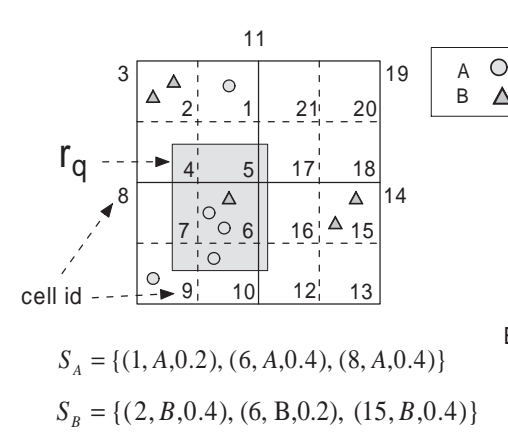

(a)

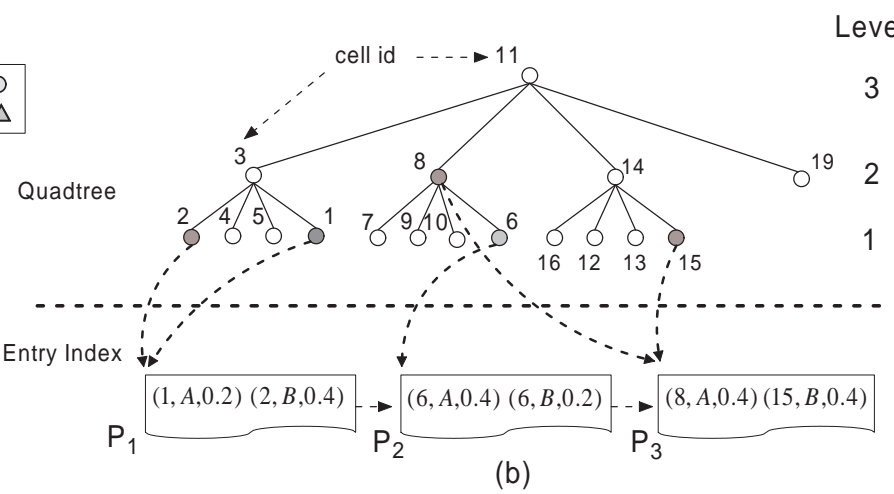

Figure 7: $U$-Quadtree

the $U$-Quadtree based range search algorithm. Section 3.3 proposes the cost model for the range search on $U$-Quadtree.

\subsection{U-Quadtree Structure}

A quadtree [12] is a space partitioning tree data structure in which a $d$-dimensional space is recursively subdivided into $2^{d}$ regions (cells). Due to its simplicity and regularity, the quadtree technique has been widely applied in many applications. In the paper, we focus on 2-dimensional space and all techniques developed can be immediately applied to higher dimensional spaces.

Given a quadtree, a summary of an object $U$ is defined as follows.

Definition $2 \quad\left(S_{U}\right)$. A summary $S_{U}$ of an object $U$ regarding a quadtree consists of a set of entries $\{e\}$ where each entry $e$ is a triplet (e.c, e.o, e.p) where e.c and e.o represent the identification (id) of the cell and the object associated with the entry, and e.p $(0<e . p \leq 1)$ is the probability mass of the instances assigned to this entry (i.e., the cell e.c). For any instance $x \in U, x$ must be assigned to exactly one cell $c$ (entry e) where $x \in c$ (e.c) and hence $\sum_{e \in S_{U}}$ e.p $=1$.

In Fig. 7(a), objects $A$ and $B$ have 5 instances each and all instances have the same occurrence probability $(0.2)$. The height of the quadtree $(h)$ is 3 and the ids of the cells are labeled. We may have $S_{A}=\{(1, A, 0.2),(6, A, 0.4),(8, A, 0.4)\}$ and $S_{B}=\{(2, B, 0.4),(6, B, 0.2),(15, B, 0.4)\}$. Note that the summary of an object is not unique as an instance $x$ can be assigned to any cell which contains $x$. For instance, an alternative of $S_{A}$ could be $\{(1, A, 0.2),(8, A, 0.8)\}$ in which 4 instances of $A$ are assigned to cell 8 on level 2 . Section 4 will investigate how to efficiently build optimal summaries.

As shown in Fig. 7(b), entries of the objects are organized based on a quadtree, named $U$-Quadtree, which consists of two parts:

- Entry Index $\left(U Q_{E}\right):$ a $B^{+}$tree used to keep entries of the objects in the secondary memory, where the key of each entry is its cell $i d$. Similar to [14], we assume the $i d$ of a cell is its Hilbert code [11] generated in a recursive way such that the cells with close spatial proximity are likely to be allocated to the same or adjacent pages in $U Q_{E}$. Particularly, a leaf node of $U Q_{E}$ is called the entry page (e.g., $P_{1}, P_{2}$ and $P_{3}$ in Fig. $7(\mathrm{~b}))$ and $f$ denotes its capacity (i.e., the maximal number of entries in an entry page).

- Quadtree $\left(U Q_{T}\right)$ : a pointer-based quadtree with height $h$. For each cell (node) $c$, let $P$ be the first entry page in which there is an entry $e$ with $e . c=c$. We keep the address of $P$ as the pointer of cell $c$. As shown in Fig. 7(b), a gray cell (node) of $U Q_{T}$ implies the pointer of the cell is not empty, i.e., there is at least one entry on it. Note that we do not need to keep a cell in $U Q_{T}$ if none of its descendent cells including itself contains any entry.

\section{2 $U$-Quadtree Based Range Search}

The following theorem indicates that we can derive the lower and upper bounds of $P_{a p p}\left(U, r_{q}\right)$, denoted by $L P_{a p p}\left(U, r_{q}\right)$ and $U P_{a p p}\left(U, r_{q}\right)$ respectively, based on the topological relations between $r_{q}$ and cells associated with $S_{U}$.

THEOREM 1. Given an object summary $S_{U}$ and a search region $r_{q}$, let $C_{1}\left(C_{2}\right)$ denote the cells in $S_{U}$ which are contained (overlapped) by $r_{q}$, we have

$$
\begin{aligned}
& L P_{a p p}\left(U, r_{q}\right)=\sum \text { e.p, where } e \in S_{U} \text { and e.c } \in C_{1} \\
& U P_{a p p}\left(U, r_{q}\right)=\sum \text { e.p, where } e \in S_{U} \text { and e.c } \in C_{1} \cup C_{2}
\end{aligned}
$$

Proof. For any point $x \in c$, we have $x \in r_{q}$ if $c$ is contained by $r_{q}$. It is immediate that $P_{a p p}\left(U, r_{q}\right) \geq \sum$ e.p where $e \in S_{U}$ and $e . c \in C_{1}$. Given a point $x \in c$, if $c$ is not contained or overlapped by $r_{q}$, then we have $x \notin r_{q}$. This implies $P_{a p p}\left(U, r_{q}\right) \leq 1-\sum$ e.p where $e \in S_{U}$ and e.c $\notin C_{1} \cup C_{2}$. Because $\sum_{e \in S_{U}}$ e.p $=1$, we have $P_{a p p}\left(U, r_{q}\right) \leq \sum$ e.p where e.c $\in C_{1} \cup C_{2}$. Therefore, the theorem holds.

EXAMPLE 2. In Fig. 7(a), given a search region $r_{q}$ (shaded region), according to Theorem 1 we have $L P_{a p p}\left(A, r_{q}\right)=0.4$ and $U P_{a p p}\left(A, r_{q}\right)=0.8$ because the cell 6 is contained by $r_{q}$ and the cell 8 is overlapped by $r_{q}$. Consequently, $A$ is pruned if the probabilistic threshold $\theta$ equals 0.9 , and $A$ is validated if $\theta=0.3$. We need to verify $A$ if $\theta=0.5$ since we cannot prune or validate the object $A$.

Algorithm 1 illustrates the details of the range search following the filtering-and-verification paradigm. Line 2 retrieves a set $\mathcal{J}$ of non-empty cells which are contained or overlapped by $r_{q}$. Let $\mathcal{D}$ denote the entry pages $\{P\}$ associated with those cells, i.e., $\mathcal{D}=\{P \mid \exists e \in P$ s.t. e.c $\in \mathcal{J}\}$. Regarding the example in Fig. $7, \mathcal{D}=\left\{P_{2}, P_{3}\right\}$. As we access entry pages in sequence, the total number of $\mathrm{I} / \mathrm{O}$ incurred in Line 4 is $|\mathcal{D}|$. According to Theorem 1, we can come up with the lower and upper bounds of the appearance probabilities of the objects. Note that any unvisited object has appearance probability zero. It is immediate that we can validate an object $U$ if $L P_{a p p}\left(U, r_{q}\right) \geq \theta$ (Line 12). Similarly an object $U$ is pruned if $U P_{a p p}\left(U, r_{q}\right)<\theta$ (Line 14). In Line 17, we only need to verify the remaining objects which are not pruned or validated during the filtering phase. 


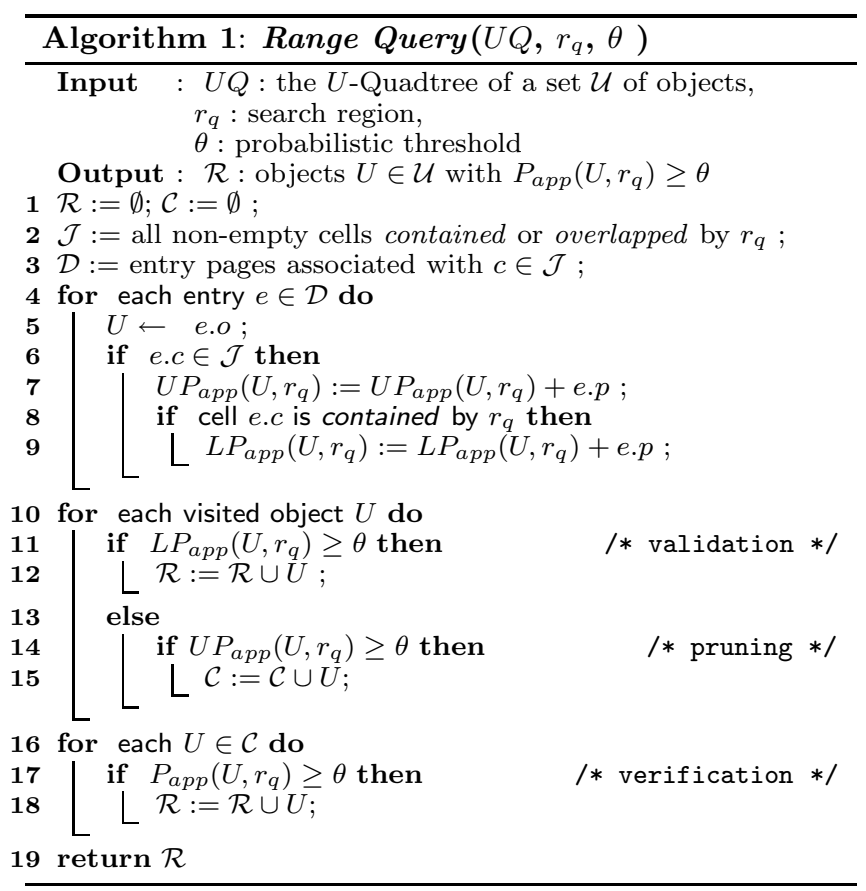

\subsection{Cost Model}

The cost of Algorithm 1 consists of four parts:

1. $C_{s}$ (Line 2), the cost to retrieve the cells contained or overlapped by $r_{q}$, which is $O\left(\frac{4^{h}-1}{3}\right)$ in the worst case.

2. $C_{\text {index }}($ Line 4$)$, the cost to load entry pages in $\mathcal{D}$, which equals $|\mathcal{D}| \times t_{\text {io }}$ where $t_{i o}$ is the delay of an I/O access.

3. $C_{\text {prob }}$ (Lines 5-9), the cost to compute lower and upper bounds of the appearance probabilities. As a hash table is employed to maintain the objects visited, $C_{\text {prob }}=$ $\sum_{U \in \mathcal{J}}\left|S_{U}\right|$ where $\mathcal{J}$ represents the visited objects and $\left|S_{U}\right|$ is the number of entries in $S_{U}$.

4. $C_{\text {verify }}$ (Line 17), the cost to verify the remaining objects in $\mathcal{C}$ where $C_{\text {verify }}=\sum_{U \in \mathcal{C}} t_{U}$, where $t_{U}$ denotes the verification cost of $U$.

The dominant costs of the range search are the index I/O $\operatorname{cost}\left(C_{\text {index }}\right)$ and the verification cost $\left(C_{\text {verify }}\right)$. In our empirical study, they contribute more than $98 \%$ of the cost for the range search. Therefore, we only consider $C_{\text {index }}$ and $C_{\text {verify }}$ in our cost model, which represent the filtering cost and verification cost of the Algorithm 1 respectively.

Suppose the query distribution $D_{\mathcal{Q}}$ is known (e.g., query $\log$ is available), below we define the containing probability and overlapping probability of a cell $c$ regarding $D_{\mathcal{Q}}$.

DEFInITION 3. Containing probability $P_{c}(c)$ and Overlapping probability $P_{o}(c)$. Suppose $r_{q}$ in Algorithm 1 is randomly chosen from a query distribution $D_{\mathcal{Q}}$, the containing (overlapping) probability, namely $P_{c}(c)\left(P_{o}(c)\right)$, of a cell $c$ is the likelihood of $c$ being contained (overlapped) by $r_{q}$.

Learn $P_{c}(c)$ and $P_{o}(c)$. Given a query distribution $D_{\mathcal{Q}}$, we can randomly retrieve $n_{q}$ search regions from $D_{\mathcal{Q}}$ and record the number of times in which the cell $c$ is contained or overlapped, denoted by $n_{c}(c)$ and $n_{o}(c)$ respectively. Then we have $P_{c}(c)=\frac{n_{c}(c)}{n_{q}}$ and $P_{o}(c)=\frac{n_{o}(c)}{n_{q}}$.
Now, we estimate the expected filtering cost and verification cost assuming the query distribution is known.

Index $\mathbf{I} / \mathbf{O}$ cost. For an entry page $P$ loaded in Algorithm 1, we say an entry $e$ in $P$ is non-redundant regarding $r_{q}$ if $e . c$ is contained or overlapped by $r_{q}$, and $e$ is redundant otherwise. For instance, in Fig. 7 (b) the entry $e(8, A, 0.4)$ $(e(15, B, 0.4))$ in $P_{3}$ is a non-redundant (redundant) entry regarding $r_{q}$. In the paper, $f_{n}$ denotes the average number of non-redundant entries for the entry pages loaded. An entry page will be loaded in the main memory if any of its associated cell is contained or overlapped by $r_{q}$. The expected number of index I/Os incurred by an entry $e$ is $\left(P_{o}(e . c)+P_{c}(e . c)\right) \times \frac{1}{f_{n}}$ since all non-redundant entries in the same entry page share the I/O latency. We can estimate the expected index I/O cost, namely $E\left(C_{\text {index }}\right)$, as follows because PDFs of the objects are mutually independent.

$$
E\left(C_{\text {index }}\right)=|\mathcal{D}| t_{i o}=\sum_{U \in \mathcal{U}} \sum_{e \in S_{U}}\left(P_{o}(e . c)+P_{c}(e . c)\right) \times \frac{t_{\text {io }}}{f_{n}}
$$

It is immediate that there is no redundant entry regarding $r_{q}$ in an entry page $P$ if all entries in $P$ are from the same cell, which is likely when the number of uncertain objects is large. Moreover, since entries are ordered by the Hilbert codes $(i d s)$ of their corresponding cells, an entry page is likely to be shared by the cells with close spatial proximity. Consequently, the number of redundant entries is small because the cells in the same page are likely to be contained or overlapped by $r_{q}$ at the same time. Therefore, we set $f_{n}$ to $f$ in the paper and Equation 3 can be rewritten as follows. Recall that $f$ is the capacity of an entry page.

$$
E\left(C_{\text {index }}\right)=\sum_{U \in \mathcal{U}} \sum_{e \in S_{U}}\left(P_{o}(e . c)+P_{c}(e . c)\right) \times \frac{t_{i o}}{f}
$$

Verification Cost. In Algorithm 1, an object $U$ will be verified if $U$ is not pruned or validated, i.e., $L P_{a p p}\left(U, r_{q}\right)<$ $\theta \leq U P_{a p p}\left(U, r_{q}\right)$. Suppose the probabilistic threshold $\theta$ is randomly chosen from $(0,1], U$ will be verified with probability $\Delta$, where $\Delta=U P_{a p p}\left(U, r_{q}\right)-L P_{a p p}\left(U, r_{q}\right)$. According to Theorem 1 , this implies that only the entries whose associated cells overlapped by $r_{q}$ contribute to the verification cost, and hence $\Delta=\sum_{e \in S_{U}} P_{o}(e . c) \times e . p$. Then based on the $P D F$ independence assumption we have the following estimation for the expected verification cost, denoted by $E\left(C_{\text {verify }}\right)$.

$$
E\left(C_{\text {verify }}\right)=\sum_{U \in \mathcal{U}} \sum_{e \in S_{U}} P_{o}(e . c) \times e . p \times t_{U}
$$

Based on Equation 4 and Equation 5 we come up with the expected cost, denoted by $E(C)$, of the range query.

$$
\begin{array}{r}
E(C)=\sum_{U \in \mathcal{U}} \sum_{e \in S_{U}}\left(\left(P_{o}(e . c)+P_{c}(e . c)\right) \times \frac{t_{i o}}{f}\right. \\
\left.+P_{o}(e . c) \times e . p \times t_{U}\right)
\end{array}
$$

Remark 1. About $t_{U}$. As shown in [21, 25], the dominant cost of the verification comes from the sampling of the $P D F$ for the continuous case or the $I / O$ cost incurred for the discrete case. The sampling cost can be estimated based on the required accuracy [21] (i.e., the number of samples), while the I/O cost can be estimated by the number of pages occupied by the object. 
In the paper, we say a $U$-Quadtree is optimal regarding the cost model if $E(C)$ is minimized. In Section 4, we investigate how to efficiently construct the optimal $U$-Quadtree regarding the cost model so that the overall cost of the range search can be minimized.

\section{BUILD $U$-QUADTREE}

A nice property of the cost model is that we can build the optimal $U$-Quadtree by optimizing the cost of the summary for each individual object. In Section 4.1, we first propose an algorithm to compute the optimal summary of an object $U$ based on the dynamic programming technique. Then Section 4.2 introduces the $U$-Quadtree maintenance algorithms. Section 4.3 discusses how to construct the $U$-Quadtree when the query distribution is unknown.

\subsection{Optimization of $S_{U}$}

For presentation simplicity, we use $F_{c}$ and $V_{c}$ to denote the filtering factor and verification factor of cell $c$ regarding $U$ where $F_{c}=\left(P_{o}(c)+P_{c}(c)\right) \times \frac{t_{i o}}{f}$ and $V_{c}=P_{o}(c) \times t_{U}$. Note that in the subsection we assume the query distribution is known and hence $P_{c}(c)$ and $P_{o}(c)$ are computed. In the paper, $\omega(e)$ denotes the cost of an entry $e$ where $\omega(e)=$ $F_{e . c}+V_{e . c} \times e . p$ if e.p $>0$, and otherwise $\omega(e)=0$. Note that $e$ is a dummy entry if $e . p=0$. The cost of a summary $S_{U}$, denoted by $\omega\left(S_{U}\right)$, is $\sum_{e \in S_{U}} \omega(e)$. We say a summary of $U$ is optimal, denoted by $S_{U}^{*}$, if it has the minimal cost among all possible summaries of $U$ regarding the quadtree.

In this subsection, we develop an efficient algorithm to construct the optimal summary of an object.

We first introduce some important properties. The following property is immediate based on the definition of the quadtree.

Property 1. For any two cells $c_{1}$ and $c_{2}$ in a $U$-Quadtree, $c_{1}$ is a descendant cell of $c_{2}$ if and only if $c_{1} \subset c_{2}$, where $c_{1} \subset c_{2}$ implies $c_{1} \subseteq c_{2}$ and $c_{1} \neq c_{2}$.

Given two cells $c_{1}$ and $c_{2}$ with $c_{1} \subset c_{2}$, if $c_{1}$ is overlapped by $r_{q}$ then $c_{2}$ is overlapped as well. This implies $V_{c_{1}} \leq V_{c_{2}}$. With similar rationale, we have $F_{c_{1}} \leq F_{c_{2}}$. Without loss of generality, we assume the following property holds.

Property 2. Given two cells $c_{1}$ and $c_{2}, V_{c_{1}}<V_{c_{2}}$ and $F_{c_{1}}<F_{c_{2}}$ if $c_{1} \subset c_{2}$.

Given a summary $S_{U}$ and a cell $c, S_{U}(c)$ denotes the instances of $U$ assigned to cell $c$ in $S_{U}$. We say a cell $c$ is empty regarding $S_{U}$ if $S_{U}(c)=\emptyset$, otherwise $c$ is occupied. For an instance $x \in U, x \rightarrow S_{U}(c)$ denotes the fact that $x$ is assigned to $c$ in $S_{U}$. Clearly, $x \rightarrow S_{U}(c)$ implies $x \in c$, but not vice versa. The following theorem implies that once an instance $x$ is assigned to a cell $c$ in $S_{U}^{*}$, all descendant cells of $c$ which contain $x$ must be empty.

TheOREm 2. Given the optimal summary $S_{U}^{*}$ and two cells $c_{1}$ and $c_{2}$ where $c_{1} \subset c_{2}$, for any two instances $x$ and $y$ where $x \in c_{1}$ and $y \in c_{1}$, we have $y \mathscr{4} S_{U}^{*}\left(c_{2}\right)$ if $x \leftrightarrow S_{U}^{*}\left(c_{1}\right)$.

Proof. The proof is by contradiction. Let $e_{1}$ and $e_{2}$ denote two entries of $S_{U}^{*}$ on $c_{1}$ and $c_{2}$ respectively. Suppose we have $x \rightarrow S_{U}^{*}\left(c_{1}\right)$ and $y \rightarrow S_{U}^{*}\left(c_{2}\right)$. This implies both cells are not empty. Let $X=\omega\left(e_{1}\right)+\omega\left(e_{2}\right)=F_{c_{1}}+V_{c_{1}} \times e_{1} . p$ $+F_{c_{2}}+V_{c_{2}} \times e_{2} . p$. Since $y \in c_{1}$, we can move $y$ from $c_{2}$ to $c_{1}$, which leads to anther summary of $U$ denoted by $S_{U}^{\prime}$.
Let $e_{1}^{\prime}$ and $e_{2}^{\prime}$ be two entries of $S_{U}^{\prime}$ on $c_{1}$ and $c_{2}$ respectively. The costs of $e_{1}^{\prime}$ and $e_{2}^{\prime}$, denoted by $Y$, is less than or equal to $F_{c_{1}}+V_{c_{1}} \times\left(e_{1} \cdot p+y_{p}\right)+F_{c_{2}}+V_{c_{2}} \times\left(e_{2} \cdot p-y_{p}\right)$. Since $c_{1} \subset c_{2}$, we have $V_{c_{1}}<V_{c_{2}}$ according to the Property 2 and hence $Y<X$. As the costs of other entries in $S_{U}^{\prime}$ remain unchanged, we have $\omega\left(S_{U}^{\prime}\right)<\omega\left(S_{U}^{*}\right)$, which is against the assumption.

Following Property is immediate based on Theorem 2.

Property 3. Let $c_{1, i}$ be a cell on level 1 , for any two instances $x$ and $y$ where $x \in c_{1, i}$ and $y \in c_{1, i}, x$ and $y$ will always be assigned to the same cell in $S_{U}^{*}$; that is, $x \rightarrow S_{U}^{*}(c)$ implies $y \rightarrow S_{U}^{*}(c)$ and vice versa.

Property 3 indicates that for the optimal summary construction, an object can be regarded as $l$ virtual instances $\left\{u_{i}\right\}$ where $u_{i}$ corresponds to the instances contained by $c_{1, i}$; that is, $u_{i}=\left\{x \mid x \in U\right.$ and $\left.x \in c_{1, i}\right\}$. In Fig. 8, 10 instances of $U$ have the same occurrence probability $(0.1)$ and they correspond to 4 virtual instances. In $S_{U}^{*}$, each virtual instance $u$ must be assigned to exactly one cell which contains $u$, and there are at most $h$ target cells for $u$. Consequently, a straightforward solution for the optimal summary construction is to enumerate all possible choices of each virtual instance, which takes $O\left(l^{h}\right)$ time where $l$ is the number of virtual instances.

To further improve the efficiency of the optimal summary construction, we develop an efficient algorithm based on the dynamic programming technique. Below, we start with a motivating example.

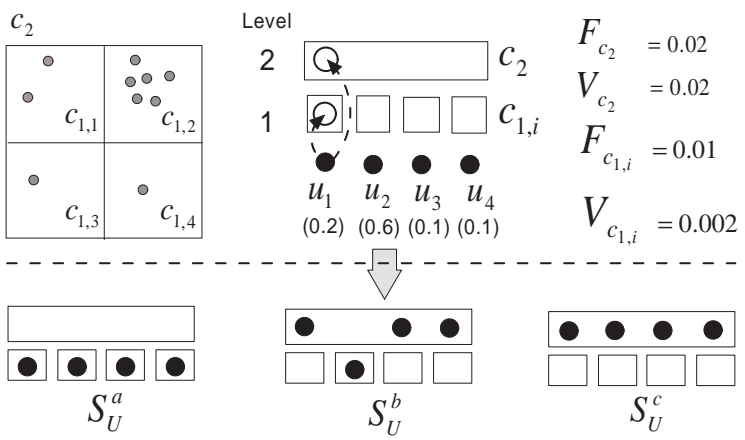

Figure 8: Motivating Example

Motivating Example. In Fig. 8, we assume that $F_{c_{2}}=$ $0.02, V_{c_{2}}=0.02, F_{c_{1, i}}=0.01$, and $V_{c_{1, i}}=0.002$ for any $i \in[1,4]$. We show three possible forms of $S_{U}$ where $S_{U}^{a}=$ $\left\{\left(c_{1,1}, 0.2\right),\left(c_{1,2}, 0.6\right),\left(c_{1,3}, 0.1\right),\left(c_{1,4}, 0.1\right)\right\}, S_{U}^{b}=\left\{\left(c_{2}, 0.4\right)\right.$, $\left.\left(c_{1,2}, 0.6\right)\right\}$ and $S_{U}^{c}=\left\{\left(c_{2}, 1.0\right)\right\}$. Note that we ignore object $i d s$ in the entries since all instances are from $U$ in this subsection. Let $e_{1}=\left(c_{2}, 0.4\right)$ and $e_{2}=\left(c_{1,2}, 0.6\right)$, we have $\omega\left(S_{U}^{b}\right)$ $=\omega\left(e_{1}\right)+\omega\left(e_{2}\right)=F_{c_{2}}+V_{c_{2}} \times 0.4+F_{c_{1,2}}+V_{c_{1,2}} \times 0.6=0.0392$. Similarly, we have $\omega\left(S_{U}^{a}\right)=0.042$ and $\omega\left(S_{U}^{c}\right)=0.04$, and hence $S_{U}^{b}$ wins out. This implies that some virtual instances can be pushed to cells at higher levels so that the cost of the summary is reduced. we use $X(X \neq \emptyset)$ to denote the set of virtual instances which are pushed to $c_{2}$, and $S_{U}^{X}$ is the corresponding summary. Compared with $S_{U}^{a}$ in which none of the virtual instance is pushed to $c_{2}$, the cost reduced by $S_{U}^{X}$, denoted by $\Delta_{X}$, is as follows.

The cost of $e_{2}^{\prime}$ is zero if $y$ is the only instance assigned to $c_{2}$ in $S_{U}^{*}$. 


$$
\begin{aligned}
\Delta_{X} & =\omega\left(S_{U}^{a}\right)-\omega\left(S_{U}^{X}\right) \\
& =\left(\sum_{u_{i} \in X}\left(F_{c_{1, i}}-\left(V_{c_{2}}-V_{c_{1, i}}\right) \times u_{i_{p}}\right)\right)-F_{c_{2}}
\end{aligned}
$$

According to Equation 7, we can see the advantage of pushing instances in $X$ to $c_{2}$ is that these instances can share the filtering cost $\left(F_{c_{2}}\right)$, and hence reduce their filtering costs if $F_{c_{2}}<\sum_{u_{i} \in X} F_{c_{1, i}}$. On the other hand, the disadvantage is that the verification cost involved is increased since $V_{c_{2}}>V_{c_{1, i}}$. We use $\Delta\left(u_{i}\right)$ to denote the gain of pushing the virtual instance $u_{i}$ to $c_{2}$ without considering the filtering cost on $c_{2}$, where $\Delta\left(u_{i}\right)=F_{c_{1, i}}-\left(V_{c_{2}}-V_{c_{1, i}}\right) \times u_{i_{p}}$. Recall that $u_{i p}$ is the occurrence probability of $u_{i}$. Particularly, we have $\Delta\left(u_{1}\right)=0.0064, \Delta\left(u_{2}\right)=-0.0008, \Delta\left(u_{3}\right)=$ $\Delta\left(u_{4}\right)=0.0082$. Let $g(X)=\sum_{u \in X} \Delta(u)$ denote the total gains of $X$, we have $\Delta_{X}=g(X)-F_{c_{2}}$. It is worthwhile to push all virtual instances in $X$ to $c_{2}$ if $g(X)>F_{c_{2}}$ because the gain can pay off the filtering cost on $c_{2}\left(F_{c_{2}}\right)$, and hence $\omega\left(S_{U}^{X}\right)<\omega\left(S_{U}^{a}\right)$. To minimize the cost of $S_{U}^{X}$ (i.e., maximize the $g(X)$ ), we should push all virtual instances $\{u\}$ with $\Delta(u)>0$; that is, $X=\left\{u_{1}, u_{3}, u_{4}\right\}$ and $g(X)=0.0228>F_{c_{2}}$, which corresponds to the summary $S_{U}^{b}$. If we have $g(X)<F_{c_{2}}$ for any $X \neq \emptyset$, none of the virtual instances should be pushed to $c_{2}$ because $\omega\left(S_{U}^{X}\right)>\omega\left(S_{U}^{a}\right)$, which is the case if we set $F_{c_{2}}=0.03$ in Fig. 8 .

Before presenting the optimal summary construction algorithm, we first introduce some important notations.

Definition $4 \quad\left(S_{c}\right.$ : summary of cell $\left.c\right)$. Let $\varphi(c)$ rep resent the cell $c$ and its descendant cells, the summary of a cell $c$, denoted by $S_{c}$, is a set of entries associated with cells in $\varphi(c)$ such that any instance contained by $c$ must be assigned to exactly one cell in $\varphi(c)$.

In the paper, $\omega\left(S_{c}\right)$ corresponds to the sum of the costs of the entries in $S_{c}$, i.e., $\omega\left(S_{c}\right)=\sum_{e \in S_{c}} \omega(e)$. Specifically, we use $S_{c}^{*}$ to represent the optimal summary of $c$ which has the smallest $\omega\left(S_{c}\right)$ value. Let $l(c)$ denote the level of a cell $c$ in $U Q_{T}, S_{c}^{*}$ corresponds to $S_{U}^{*}$ if $l(c)=h$.

We say an instance $x$ is pushed up above $c$ if $x \in c$ and $x$ is assigned to a cell at higher level than $c$. For instance, in Fig. $8 u_{1}$ is pushed up above $c_{1,1}$ in $S_{U}^{b}$ and $S_{U}^{c}$. The following property indicates that all instances pushed up above $c$ in $S_{U}^{*}$ must be assigned to the same cell. The correctness of the Property is immediate based on Theorem 2.

Property 4. If an instance $x$ is pushed up above $c$ in $S_{U}^{*}$, i.e., $x \in c$ and $x \rightarrow S_{U}^{*}\left(c_{j}\right)$ where $c \subset c_{j}$, then $y \rightarrow S_{U}^{*}\left(c_{j}\right)$ for any instance $y$ pushed up above $c$ in $S_{U}^{*}$.

Property 4 is essential to our optimal summary construction algorithm, because it suggests that for each $c$ we can evaluate the possible gain of pushing up above $c$ against its individual ancestor cell, i.e., cells $\left\{c_{j}\right\}$ with $c \subset c_{j}$ according to Property 1. Consequently, for each ancestor cell $c_{j}$ of $c\left(c \subset c_{j}\right)$, we use $S_{c, c_{j}}$ to denote the summary of $c$ regarding $c_{j}$ which is defined as follows.

DEFINITION $5 \quad\left(S_{c, c_{j}}\right.$ : summary of $c$ regarding $\left.c_{j}\right)$. The summary of a cell c regarding $c_{j}$ where $c \subseteq c_{j}$, denoted by $S_{c, c_{j}}$, is a set of entries associated with the cells in $\varphi(c)$ and $c_{j}$ such that any instance contained by $c$ must be assigned to exactly one cell in $\varphi(c)$ or $c_{j}$.
In the paper, $\omega\left(S_{c, c_{j}}\right)$ corresponds to the total costs of the entries in $S_{c, c_{j}}$ without considering the filtering cost of the entry on $c_{j}$ (denoted by $e_{c_{j}}$ ), i.e., $\omega\left(S_{c, c_{j}}\right)=\left(\sum_{e \in\left(S_{c, c_{j}}-e_{c_{j}}\right)}\right.$ $\omega(e))+V_{c_{j}} \times e_{c_{j}} . p$. Moreover, we use $\Delta\left(S_{c, c_{j}}\right)$ to denote the gain of $c$ regarding $c_{j}$, where $\Delta\left(S_{c, c_{j}}\right)=\omega\left(S_{c}^{*}\right)-\omega\left(S_{c, c_{j}}\right)$. In the paper, $S_{c, c_{j}}^{*}$ denotes a set of entries with the smallest $\omega\left(S_{c, c_{j}}\right)$ value (i.e., the largest $\Delta\left(S_{c, c_{j}}\right)$ value), which is named the optimal summary of $c$ regarding $c_{j}$. Recall that the main difference between $S_{c, c_{j}}$ and $S_{c}$ is that the instances contained by $c$ can be pushed to $c_{j}$ in $S_{c, c_{j}}$ and the cost of $S_{c, c_{j}}\left(\omega\left(S_{c, c_{j}}\right)\right)$ does not include the filtering cost on $c_{j}$. Note that we have $\Delta\left(S_{c, c_{j}}^{*}\right) \geq 0$ because $S_{c}$ is a special case of $S_{c, c_{j}}$ where none of the instances contained by $c$ is assigned to $c_{j}$.

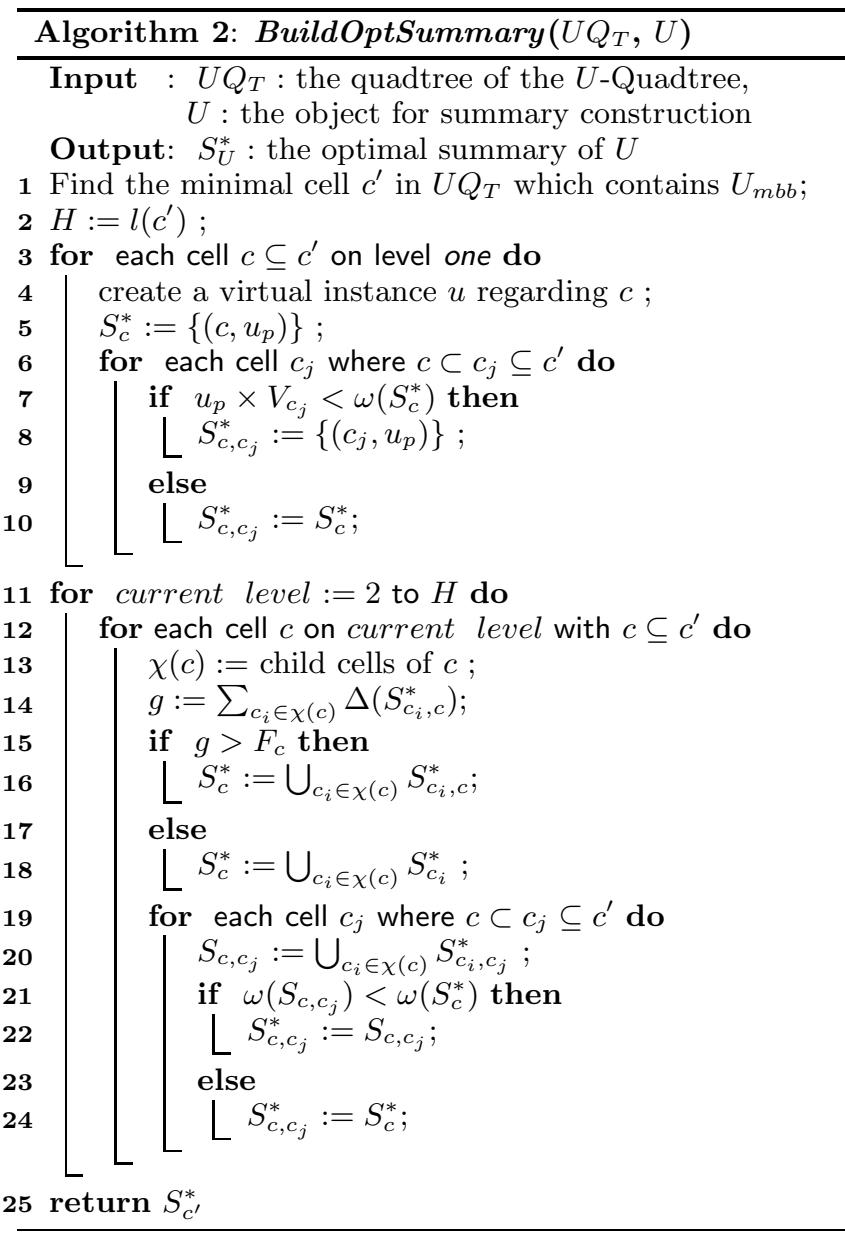

Build Optimal Summary. Algorithm 2 illustrates the construction of $S_{U}^{*}$ based on the dynamic programming technique. Line 1 identifies the minimal cell $c^{\prime}$ which contains the $\mathrm{MBB}$ of the object. In Lines 3-10, we create virtual instances for all descendant cells of $c^{\prime}$ on the first level, as well as their corresponding optimal summaries. Lines 11-24 show how to build related optimal summaries of a cells $c$ with $l(c)>1$. Let $\chi(c)$ denote the child cells of $c$ (Line 13). In Line 14, we first compute the total gains, denoted by $g$, of the child cells (i.e., $\chi(c)$ ) regarding $c$. If the gains can

We have $e_{c_{j}} \cdot p=0$ (i.e., $e_{c_{j}}$ is a dummy entry) if none of the instances is assigned to $c_{j}$ in $S_{c, c_{j}}$. 
pay off the filtering cost on cell $c$ (i.e., $g>F_{c}$ ), we come up with $S_{c}^{*}$ by merging its child optimal summaries regarding $c$ (Line 16), otherwise $S_{c}^{*}$ consists of the optimal summaries of its child cells (Line 18). Lines 19-24 compute the optimal summaries of $c$ regarding its ancestor cells. Specifically, for each ancestor cell $c_{j}$ of $c$ with $c_{j} \subseteq c^{\prime}, S_{c, c_{j}}$ is the merged result of the optimal summaries of its child cells regarding $c_{j}$ (Line 20). If there is no gain compared with $S_{c}^{*}$ (i.e., $\left.\omega\left(S_{c, c_{j}}\right) \geq \omega\left(S_{c}^{*}\right)\right), S_{c, c_{j}}^{*}$ is set to $S_{c}^{*}$ (Line 24). Otherwise, $S_{c, c_{j}}$ is the optimal summary of $c$ regarding $c_{j}$ (Line 22).

Example 3. In Fig. 8, we have $\omega\left(S_{c_{1,1}}^{*}\right)=0.01+0.002 \times$ $0.2=0.0104, \omega\left(S_{c_{1,2}}^{*}\right)=0.0112$, and $\omega\left(S_{c_{1,3}}^{*}\right)=\omega\left(S_{c_{1,4}}^{*}\right)=$ 0.0102. Meanwhile, since $V_{c_{2}} \times u_{1} . p=0.004<\omega\left(S_{c_{1,1}}^{*}\right)$, we have $\omega\left(S_{c_{1,1}, c_{2}}^{*}\right)=0.004, \Delta\left(S_{c_{1,1}, c_{2}}^{*}\right)=\omega\left(S_{c_{1,1}}^{*}\right)-\omega\left(S_{c_{1,1}, c_{2}}^{*}\right)$ $=0.0064$ and $S_{c_{1,1}, c_{2}}^{*}=\left\{\left(c_{2}, 0.2\right)\right\}$. Similarly, we have $\omega\left(S_{c_{1,3}, c_{2}}^{*}\right)=\omega\left(S_{c_{1,4}, c_{2}}^{*}\right)=0.002, \Delta\left(S_{c_{1,3}, c_{2}}^{*}\right)=\Delta\left(S_{c_{1,4}, c_{2}}^{*}\right)$ $=0.0082$ and $S_{c_{1,3}, c_{2}}^{*}=S_{c_{1,4}, c_{2}}^{*}=\left\{\left(c_{2}, 0.1\right)\right\}$. Since there is no gain by pushing $u_{2}$ to $c_{2}$ as $0.6 \times V_{c_{2}}>\omega\left(S_{c_{1,2}}^{*}\right)$, we have $\omega\left(S_{c_{1,2}, c_{2}}^{*}\right)=\omega\left(S_{c_{1,2}}^{*}\right)=0.0112$, and hence $\Delta\left(S_{c_{1,2}, c_{2}}^{*}\right)=0$ and $S_{c_{1,2}, c_{2}}^{*}=S_{c_{1,2}}^{*}=\left\{\left(c_{1,2}, 0.6\right)\right\}$. In Algorithm $2, g=$ $\sum_{c \in \chi\left(c_{2}\right)} \Delta\left(S_{c, c_{2}}^{*}\right)=0.0228$ and $g>F_{c_{2}}=0.02$. Therefore, we have $S_{U}^{*}=S_{c_{2}}^{*}=\bigcup_{c \in \chi\left(c_{2}\right)} S_{c, c_{2}}^{*}=\left\{\left(c_{1,2}, 0.6\right),\left(c_{2}, 0.4\right)\right\}$ and $\omega\left(S_{U}^{*}\right)=0.0392$.

Correctness. With similar argument in Theorem 2, regarding all instances assigned to the cells above $c^{\prime}$, we can move them to $c^{\prime}$ to reduce the cost since all instances are contained by $c^{\prime}$. Therefore, we only need to consider the cells in $\varphi\left(c^{\prime}\right)$ during the optimal summary construction.

The following proof is by induction on the levels of $U$ Quadtree. The correctness of the optimal summaries of the cells on the first level is immediate based on their definitions. Suppose Algorithm 2 is correct regarding cells on the levels lower than $k$, then for a cell $c$ on level $k$, we first show the correctness of its optimal summary $S_{c}^{*}$.

Clearly, the cell $c$ is either empty or occupied in $S_{c}^{*}$. Let $S_{c}^{1}$ denote the merged result of the optimal summaries of its child cells, i.e., $S_{c}^{1}=\bigcup_{c_{i} \in \chi(c)} S_{c_{i}}^{*}$. $S_{c}^{1}$ is a summary of $c$ because, for any instance $x \in c$, there is exactly one child cell $c_{i} \in \chi(c)$ in which $x \in c_{i}$ and hence $x$ is assigned to exactly one cell in $S_{c}^{1}$. Moreover, we have $\omega\left(S_{c}^{1}\right)=\sum_{c_{i} \in \chi(c)} \omega\left(S_{c_{i}}^{*}\right)$. Then we show $S_{c}^{1}$ is the optimal summary when $c$ is empty. The proof is by contradiction. Suppose there is another summary $S_{c}^{2}$ with $\omega\left(S_{c}^{2}\right)<\omega\left(S_{c}^{1}\right)$, where $S_{c}^{2}=\bigcup_{c_{i} \in \chi(c)} S_{c_{i}}^{2}$ and $S_{c}^{2}(c)=\emptyset$, then there is a summary $S_{c_{i}}^{2}$ where $c_{i} \in \chi(c)$ such that $\omega\left(S_{c_{i}}^{2}\right)<\omega\left(S_{c_{i}}^{*}\right)$ because $\omega\left(S_{c}^{2}\right)=\sum_{c_{i} \in \chi(c)} \omega\left(S_{c_{i}}^{2}\right)$. This is against our assumption since the level of $c_{i}$ is lower than $k$ and $S_{c_{i}}^{*}$ is the optimal summary of $c_{i}$. Therefore, $S_{c}^{1}$ is the optimal summary when $c$ is empty.

If $c$ is occupied in $S_{c}^{*}$, some instances must be pushed to $c$ in $S_{c}^{*}$. Let $S_{c}^{3}$ denote the merged result of the optimal summaries of the child cells regarding $c$, i.e., $S_{c}^{3}=\bigcup_{c_{i} \in \chi(c)} S_{c_{i}, c}^{*}$. $S_{c}^{3}$ is a summary of $c$ because any instance contained by $c$ is contained by exactly one of its child cell, say $c_{i}$, and any instance $x \in c_{i}$ must be assigned to exactly one cell in $S_{c_{i}, c}^{*}$. With similar rational to the above case where $c$ is empty, we

Recall that we do not include the filtering cost on $c\left(F_{c}\right)$ when computing the cost of the optimal summary of each child cell regarding $c$ (i.e., $\omega\left(S_{c_{i}, c}^{*}\right)$ for each $\left.c_{i} \in \chi(c)\right)$.

Entries with the same cell $i d$ will be merged by accumulating their probability values. can prove that $S_{c}^{3}$ has the lowest cost among the summaries of $c$ in which $c$ is occupied. $g>F_{c}$ in Line 15 of Algorithm 2 implies that $\omega\left(S_{c}^{3}\right)<\omega\left(S_{c}^{1}\right)$; that is, it is worthwhile to occupy the cell $c$ by pushing some instances to $c$ because this can pay off the filtering cost of $c$. Consequently, $S_{c}^{3}$ is the optimal summary when $c$ is occupied and hence the correctness of the optimal summaries of the cells on level $k$ holds.

The correctness of the optimal summary of $c$ regarding its ancestor cells can be obtained with similar rationale, we omit the details of the proof due to space limits.

Cost Analysis. In Algorithm 2, it takes $O(h)$ time to locate $c^{\prime}$ where $h$ is the height of the $U$-Quadtree. It takes $O(h m)$ time to create the virtual instances in a top-down fashion where $m$ is the number of the instances in $U$. As to each cell $c$, it costs $O(h)$ time to compute related optimal summaries $\left(S_{c}^{*}\right.$ and $S_{c, c_{j}}^{*}$ where $\left.c \subset c_{j}\right)$. Note that it takes $O(1)$ time to merge child summaries and calculate related costs at Line 16, 18 and 20 because the child summaries share at most one cell (e.g., cell $c$ in Line 16). Therefore, the time complexity of Algorithm 2 is $O\left(h \times m+h \times \frac{4^{h}-1}{3}\right)$ in the worst case since the number of cells in $U Q_{T}$ is bounded by $\frac{4^{h}-1}{3}$. For a cell $c$ on level $s$, there are at most $4^{s-1}$ entries for each optimal summary since $c$ has at most $4^{s-1}$ descendant cells on the first level. There are at most $4^{h-s}$ cells on level $s$ and we only need to keep at most $h-s+1$ related optimal summaries for each cell. Therefore, the space usage for each level is $O\left(4^{h-1} \times h\right)$ in the worst case. Since in Algorithm 2 we do not need to keep the summaries of the child cells once the optimal summaries computation of a cell is finished, the space complexity of Algorithm 2 is $O\left(4^{h-1} \times h\right)$.

Addressing three factors in Section 1. The final part of this subsection shows that three factors mentioned in Section 1 are carefully addressed in Algorithm 2. The resources allocated to an object are measured by the number of entries in its optimal summary. For an object with large uncertain region size, the number of virtual instances is large and hence more resources (i.e., the number of entries in $S_{U}^{*}$ ) may be allocated. The instances in the sparse part of the $P D F$ tends to be pushed to the cells on higher levels in $U$-Quadtree because the gain of "pushing up" is more significant than that of the instances from the dense part. Therefore, more resources are allocated to the dense part of an object. With same rationale, the instances of the objects with high verification cost tend to stay on the lower level cells, and hence lead to a large summary size.

\subsection{Optimal $U$-Quadtree Construction}

According to the cost model in Section 3, the $U$-Quadtree is optimal if all summaries of the objects are optimal because $E(C)=\sum_{U \in \mathcal{U}} \omega\left(S_{U}\right)$. Therefore, we can easily come up with the optimal $U$-Quadtree construction algorithm by updating $U Q_{T}$ and $U Q_{E}$ based on the entries in $S_{U}^{*}$.

Algorithm 3 illustrates how to insert an object into the $U$ Quadtree. We first create the optimal summary of $U$ based on Algorithm 2 (Line 1). Then each entry of $S_{U}^{*}$ is inserted to the entry index of $U$-Quadtree (Line 2-8). The procedure is the same as the insertion of $B^{+}$tree except that we need to maintain page links of the cells (Lines 6 and 8).

Cost Analysis. Let $n$ and $n_{e}$ denote the number of the objects in $\mathcal{U}$ and the average number of entries in the optimal summaries, respectively. According to the analysis of Algo-

$\overline{\text { Suppose } x \in c \text {, it takes } O(1)}$ time to identify the child cell of $c$ which contains $x$. 


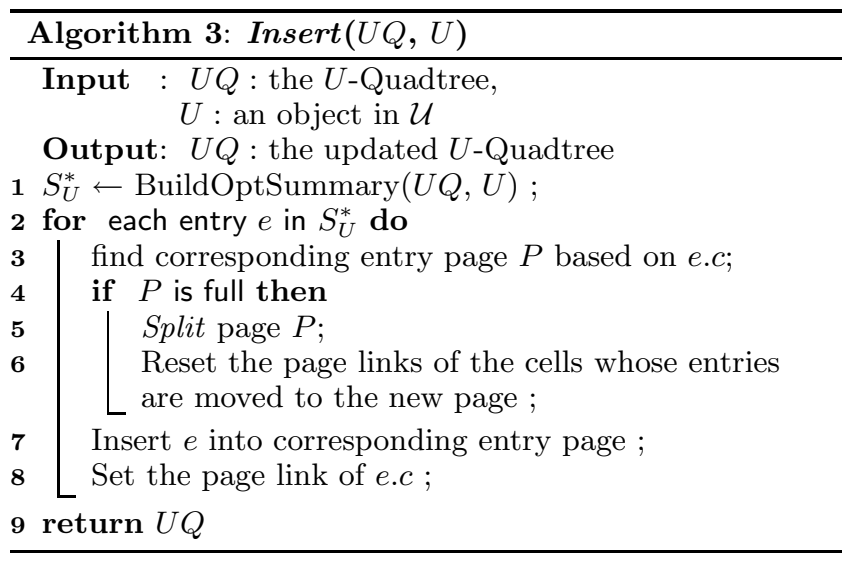

rithm 2 , the space complexity of the optimal $U$-Quadtree construction algorithm is $O\left(n \times n_{e}+4^{h-1} \times h\right)$. The dominant cost of Algorithm 3 is the summary construction and $B^{+}$Tree $\left(U Q_{E}\right)$ maintenance. According to [10], the amortized cost of the insertion for $B^{+}$Tree is $O\left(\log _{f} n \times n_{e}\right)$. Consequently, the time complexity of our index construction algorithm is $O\left(n \times \frac{4^{h}-1}{3} \times h+n \times n_{e} \times \log _{f}\left(n \times n_{e}\right)\right)$.

Deletion. The deletion of an object $U$ from $U$-Quadtree is straightforward. Firstly we find the cells which are contained or overlapped by $U_{m b b}$. Then the entries associated with the cells are removed from $U Q_{E}$. Same as the insertion, we also need to maintain the page links of the cells.

\subsection{Unknown Query Distribution}

When the distribution of the query load is unknown, it is intuitive to construct the $U$-Quadtree with query uniform assumption. This is arguably the best choice since that, without any priori knowledge, we should assume that all cells at the same level of the $U$-Quadtree have the same chance to be contained or overlapped by the search region. In the experiments, we construct the $U$-Quadtree based on the query uniform assumption and it is shown that our technique can significantly outperform the competitors against various query distributions. Particularly, we assume each query region is a square whose center is uniformly distributed in the space $[0,1]^{d}$ and the length $l$ is randomly chosen from $[0,1]$. With similar argument in [22], we have $P_{c}(c)+P_{o}(c)=$ $\min \left((l+w)^{d}, 1.0\right)$ where $w$ is the length of cell $c$. Similarly, $P_{o}(c)=(l+w)^{d}-(l-w)^{d}$ if the $w<l$; Otherwise, $P_{o}(c)=\min \left((l+w)^{d}, 1.0\right)$.

\section{PERFORMANCE EVALUATION}

We present results of a comprehensive performance study to evaluate the efficiency and scalability of the proposed techniques in the paper. Following algorithms are evaluated.

$U$-Tree The $U$-Tree technique presented in [21]. Note that the $R$-tree based technique is a special case of $U$-Tree where the catalog size equals one. As shown in [21], the $R$-tree technique is significantly outperformed by $U$ Tree. So we exclude $R$-tree technique in our empirical study.

$U I$-Tree The $U I$-Tree technique proposed in [25].

$U P$ The pivot indexing technique proposed in [3].
$U D$-Tree The $U$-Quadtree technique. By default, the query uniform assumption in Section 4.3 is used to construct $U$-Quadtree for comparison fairness.

Datasets. Three real spatial datasets, $C A, L B$ and $U S$, are employed to represent the centers of the uncertain regions. They contain $62 K, 53 K$ and $200 K$ 2-dimensional points representing locations in Los Angeles, Long Beach and the United States respectively which are available at http://www.census.gov/geo/www/tiger and $C A$ is the default dataset. We also generate synthetic dataset where dimensionality various from 2 to 4 , named $2 D, 3 D$ and $4 D$ respectively. There are $200 K$ points in each synthetic dataset and they are uniformly distributed. All dimensions are normalized to domain [0,10000]. In our experiment, the uncertain region of the uncertain object is a circle or sphere with expected radius $r_{u}$ varying from 100 to 300 with default value 200 for 2-dimensional data. $r_{u}$ is set to 600 and 1200 for 3 and 4 dimensional data respectively. For a given $r_{u}$, the radius of the objects are randomly chosen between 0 and $2 \times r_{u}$. Suppose the $P D F$ of an object is described by $m$ instances which follow two popular distributions Normal and Uniform, where the expected $m$ varies from 200 to 1000 with default value 400. Given $m$, the number of instances for each object uniformly distributes between 0 and $2 m$. The Normal serves as default distribution with standard deviation $\frac{r_{u}}{2}$. There are totally around $24.8 M$ instances for the default dataset $(C A)$ and the maximal number of instances is $80 M$ in $U S$ dataset.

Workload. A workload for the range query consists of 200 queries in our experiments. Same as [21, 25], the region of a range query $r_{q}$ is a circle or sphere with radius $\gamma$ (query distance). By default, $\gamma$ is uniformly distributed between 500 and 1500 in 2-dimensional space, and $\gamma$ is set to 1900 and 3500 in 3 and 4 dimensional space respectively. We use $Q(C A)$ to denote the queries whose centers are generated from the centers of the $C A$ datasets. Similarly, we have $Q(L B)$ and $Q(U S)$. By default, the query distribution follows the underlying target data distribution. For instance, $Q(C A)$ is the default query distribution for $C A$ dataset. We also create synthetic query distributions whose centers follow the uniform, anti-correlated and correlated distributions based on the generator from [7], denoted by $Q(E), Q(A)$ and $Q(C)$ respectively. Meanwhile, we randomly choose the probabilistic threshold $\theta \in(0,1]$ for each query. The average response times are recorded to evaluate the overall performance of the range searching algorithms. We also record the average number of disk accesses (index I/O and data I/O), candidate objects and false positives.

All algorithms proposed in this paper are implemented in standard C++ with STL library support and compiled with GNU GCC. Experiments are run on a PC with Intel Xeon $2.40 \mathrm{GHz}$ dual CPU and $4 \mathrm{G}$ memory running Debian Linux. The disk page size is fixed to 4096 bytes and the capacity of the entry page $(f)$ is set to 512 .

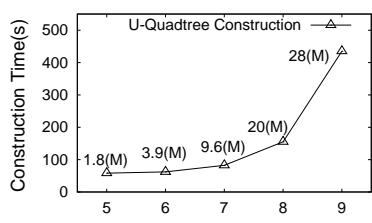

Figure 9: Diff. $h$

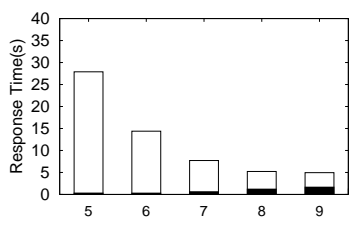

Figure 10: Diff. $h$
In order to achieve the best overall performance, the catalog size of $U$-Tree [21] is set to 8, 10 and 12 for 2, 3 and 


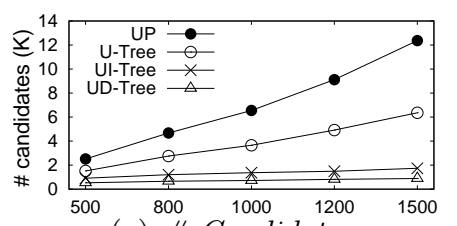

(a) \# Candidates

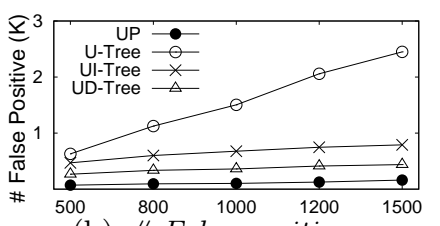

(b) \# False positives

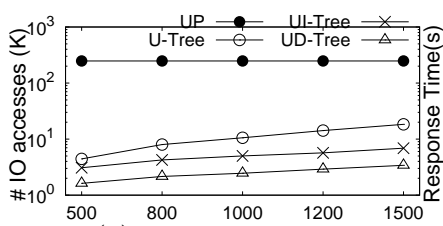

(c) \# IO accesses

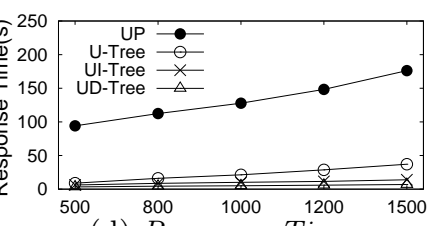

(d) Response Time

Figure 11: Diff. $\gamma$

4-dimensional dataset respectively. Similarly, the merge factor $m$ of $U I$-Tree [25] is set to 8,4 and 3 respectively. As suggested in [3], we set the histogram size $l$ to 100 and there are $10 \times d$ pivot points for $d$-dimensional dataset. Table 3 lists parameters which may have an impact on our performance study. In our experiments, all parameters use default values unless otherwise specified.

\begin{tabular}{|c|l|}
\hline Notation & Definition (Default Values) \\
\hline$h$ & height of $U$-Quadtree $(8)$ \\
\hline$\gamma$ & query distance $(1000)$ \\
\hline$r_{u}$ & radius of uncertain object region $(200)$ \\
\hline$n$ & number of uncertain objects $(62 K)$ \\
\hline$m$ & number of instances per object $(400)$ \\
\hline$\theta$ & probabilistic threshold $(\in(0,1])$ \\
\hline
\end{tabular}

Table 3: System Parameters

\subsection{Construction of $U$-Quadtree}

For comparison fairness, in the experiments we construct the $U$-Quadtree based on the uniform assumption of the query load as discussed in Section 4.3. We set $t_{i o}=1$ and set $t_{U}$ to the number of pages occupied by the object $U$ since we only need to know the ratio of $\frac{t_{U}}{t_{i o}}$ for the optimal $U$-Quadtree construction, instead of exact $t_{i o}$ and $t_{U}$ values.

Fig. 9 shows the construction times on $C A$ dataset where $h$ varies from 5 to 9 . As expected, the construction time and index size grow with $h$. The index size is $20 M$ when $h=8$, which is $1.3 \%$ of the dataset. The index size of $U$-Tree, $U I$ Tree and $U P$-Index are $13 M, 34 M$ and $969 M$ respectively. The construction time of $U D$-Tree is 155 second when $h=8$, and it takes 120, 332, 1152 seconds for $U$-Tree, $U I$-Tree and $U P$ respectively.

Fig. 10 reports the range query response time where the solid bar and empty bar represent the filtering time and verification time respectively. It is interesting to see that the performance of the algorithm becomes stable once $h$ is sufficiently large $(h \geq 8)$. This is because the instances of the objects can be "pushed up" to a proper level by the index construction algorithm. In the following experiments, we set $h$ to 8,6 and 4 for 2,3 and 4 dimensional data respectively.

\subsection{Evaluate Range Query}

In this subsection, we evaluate the performance of the algorithms for range searching.

Impact of query distance $(\gamma)$. Fig. 11 reports the average query response time, the average number of candidates, false positives and disk accesses of four algorithms ( $U$-Tree, $U I$-Tree, $U P$ and $U D$-Tree) against query distance $\gamma$. Recall that, by default the query distribution is obtained from the centers of the underlying target objects. Therefore $Q(C A)$ is the query distribution. Results show that although $U P$ Algorithm has the smallest number of false positives, the candidate size is large because $U P$-Index Algorithm cannot validate any object. Moreover, the number of $\mathrm{I} / \mathrm{O}$ accesses is also large since the whole index is scanned for the object pruning. Therefore, the overall performance of $U P$ Index Algorithm is not competitive compared with other algorithms and hence we exclude $U P$-Index Algorithm in the following experiments. Fig. 11 shows that $U D$-Tree Algorithm always outperforms $U I$-Tree and $U$-Tree Algorithms.

Impact of query distribution. Fig. 12 reports the response time of the algorithms on $C A$ dataset where various query distributions are employed. Particularly, the $U D$ Tree(OPT) Algorithm constructs the $U$-Quadtree based on each corresponding query distribution (i.e., query distribution is known). Recall that the query uniform assumption in Section 4.3 is employed for $U D$-Tree Algorithm. It is shown that $U D$-Tree(OPT) Algorithm can make some performance improvement compared with $U D$-Tree Algorithm. Results shows that $U D$-Tree Algorithm significantly outperforms $U$ Tree and $U I$-Tree Algorithms under all query distributions, which implies that constructing $U$-Quadtree based on query uniform assumption is a good choice in practise when the query distribution is unknown.

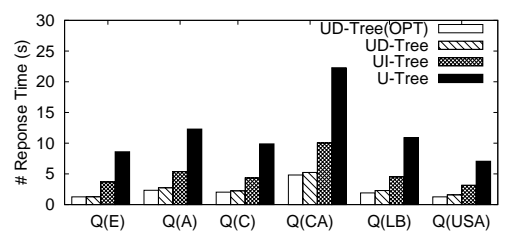

Figure 12: Diff. Query Distributions

Impact of datasets. Fig. 13 reports the number of I/O accesses and the query response time of three algorithms against $C A, L B, U S$ and $3 D$ datasets. Results show that $U D$-Tree Algorithms ranks first under all datasets.

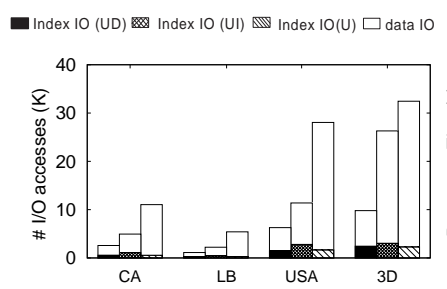

(a) $I / O$ (b) Response Time

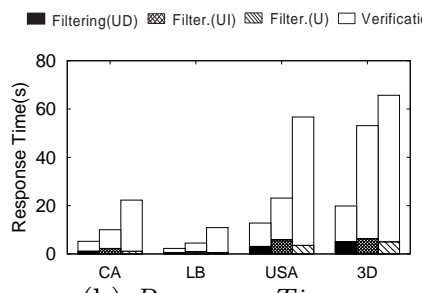

Figure 13: Performance vs diff. datasets

Impact of other parameters. We also study the impact of other parameters which may potentially affect the performance of the algorithms. Specifically, Fig. 14(a), Fig. 14(b), Fig. 14(c) and Fig. 14(d) investigate the scalability of the algorithms against $d$ (dimensionality), $r_{u}$ (uncertain region size), $m$ (instance size) and $n$ (object size) respectively. As expected, Fig. 14(a) shows that the performances of three algorithms degrade rapidly against dimensionality. Nevertheless, $U D$-Tree Algorithm is more scalable than $U I$-Tree and

Note that $U P$-Index focus on supporting the range search on general metric space, which cannot be achieved by $U$ Tree, $U I$-Tree and $U$-Quadtree techniques. 


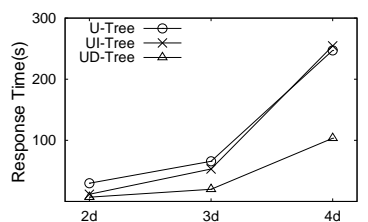

(a) Diff. d

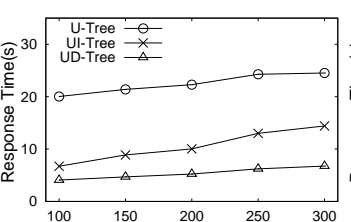

(b) Diff. $r_{u}$

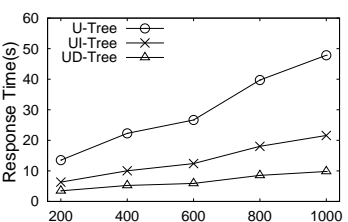

(c) Diff. $m$

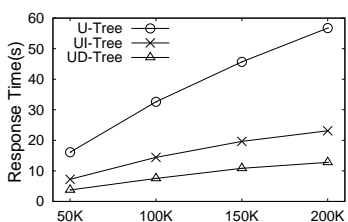

(d) Diff. $n$

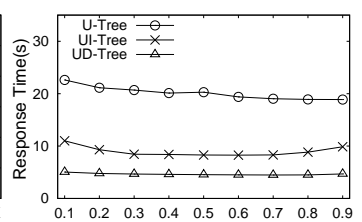

(e) Diff. $\theta$

Figure 14: Impact of various parameters

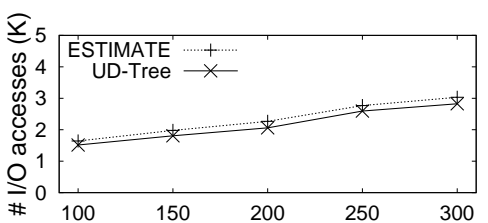

Figure 15: Accuracy Evaluation

$U$-Tree Algorithms, and $U D$-Tree Algorithm always outperforms other algorithms under all settings. Note that in Fig. 14(d) we randomly choose $50 K, 100 K$ and $150 K$ objects from $U S$ dataset. Finally, Fig. 14(e) shows that the performances of the algorithms are not sensitive to the probabilistic threshold $\theta$.

\subsection{Accuracy of the Cost Model}

We evaluate the accuracy of the cost model by estimating the number of I/Os in the experiments. Particularly, in the cost model (Equation 6 ) we set $t_{i o}$ and $t_{U}$ to 1 and the number of pages the object $U$ occupied respectively. Then $E(C)$ corresponds to the expected number of I/O accessed. Fig. 15 verifies the accuracy of the cost model on $C A$ dataset where $r_{u}$ varies from 100 to 300, and search regions are squares with length 4000 . The total number of $\mathrm{I} / \mathrm{O}$ accesses are predicated, and results show the proposed analytical model is quite accurate, the relative error usually being around $8 \%$.

\section{CONCLUSION AND FUTURE WORK}

To address the uncertainty in various applications, we have developed an effective indexing technique to support range search on multi-dimensional uncertain objects. Based on some insights of the range search we build a cost model which carefully considers various factors which may impact the performance of the range query. An effective and efficient index construction algorithm is proposed based on the cost model. Our experiments convincingly demonstrate the effectiveness and efficiency of our indexing techniques. As a future work, we will investigate how to extend our indexing technique to tackle the correlation among the multidimensional uncertain objects.

Acknowledgement. Ying Zhang is supported by ARC DP110104880 and UNSW ECR grant PS27476. Wenjie Zhang is supported by ARC DP120104168 and ARC DE12010 2144. Xuemin Lin is supported by ARC DP0987557, DP110102937 and DP120104168.

\section{REFERENCES}

[1] P. K. Agarwal, S.-W. Cheng, Y. Tao, and K. Yi. Indexing uncertain data. In PODS, 2009.

[2] C. Aggarwal and P. Yu. On high dimensional indexing of uncertain data. In ICDE 2008.

[3] F. Angiulli and F. Fassetti. Indexing uncertain data in general metric space. TKDE, to appear.
[4] G. Beskales, M. A. Soliman, and I. F. Ilyas. Efficient search for the top-k probable nearest neighbors in uncertain databases. PVLDB, 1(1), 2008.

[5] C. Böhm, M. Gruber, P. Kunath, A. Pryakhin, and M. Schubert. Prover: Probabilistic video retrieval using the Gauss-tree. In ICDE $200 \%$.

[6] C. Böhm, A. Pryakhin, and M. Schubert. Probabilistic ranking queries on Gaussians. In SSDBM 2006.

[7] S. Börzsönyi, D. Kossmann, and K. Stocker. The skyline operator. In ICDE, 2001.

[8] J. Chen and R. Cheng. Efficient evaluation of imprecise location-dependent queries. In ICDE, 2007.

[9] R. Cheng, Y. Xia, S. Prabhakar, R. Shah, and J. S. Vitter. Effcient indexing methods for probabilistic threshold queries over uncertain data. In VLDB 2004.

[10] D. Comer. The ubiquitous b-tree. ACM Comput. Surv., 11(2):121-137, 1979.

[11] C. Faloutsos. Gray codes for partial match and range queries. IEEE Trans. Software Eng., 1988.

[12] R. A. Finkel and J. L. Bentley. Quad trees: A data structure for retrieval on composite keys. Acta Inf., 1974.

[13] E. Frentzos, K. Gratsias, and Y. Theodoridis. On the effect of location uncertainty in spatial querying. TKDE, 21(3):366 -383, 2009.

[14] G. R. Hjaltason and H. Samet. Speeding up construction of pmr quadtree-based spatial indexes. VLDB J., 2002.

[15] H. Kimura, S. Madden, and S. B. Zdonik. Upi: A primary index for uncertain databases. PVLDB, 2010.

[16] H.-P. Kriegel, P. Kunath, M. Pfeifle, and M. Renz. Probabilistic similarity join on uncertain data. In DASFAA, 2006.

[17] X. Lian and L. Chen. A generic framework for handling uncertain data with local correlations. PVLDB, 2010

[18] Y. Ma, D. V. Kalashnikov, and S. Mehrotra. Toward managing uncertain spatial information for situational awareness applications. TKDE, 20(10), 2008.

[19] J. H. Schiller and A. Voisard. Location-based Services. Morgan Kaufmann, 2004.

[20] S. Singh, C. Mayfield, S. Prabhakar, R. Shah, and S. E. Hambrusch. Indexing uncertain categorical data. In ICDE, 2007.

[21] Y. Tao, X. Xiao, and R. Cheng. Range search on multidimensional uncertain data. ACM Trans. Database Syst., 32(3), 2007.

[22] Y. Theodoridis and T. K. Sellis. A model for the prediction of r-tree performance. In PODS, 1996.

[23] T. T. L. Tran, C. Sutton, R. Cocci, Y. Nie, Y. Diao, and P. J. Shenoy. Probabilistic inference over rfid streams in mobile environments. In ICDE, 2009.

[24] M. Zhang, S. Chen, C. S. Jensen, B. C. Ooi, and Z. Zhang. Effectively indexing uncertain moving objects for predictive queries. PVLDB, 2009.

[25] Y. Zhang, X. Lin, W. Zhang, J. Wang, and Q. Lin. Effectively indexing the uncertain space. TKDE, 2010. 